\title{
Episodic facial edema as a presentation of pleomorphic adenoma - case report
}

\author{
Martins $\mathrm{F}^{1,2}$, Pereira $\mathrm{E}^{1,2}$ \\ ${ }^{1}$ Internal Medicine Department, Centro Hospitalar São João, Porto, Portugal \\ ${ }^{2}$ Faculty of Medicine, University of Porto, Portugal
}

\begin{abstract}
Peripheral edema is a common complaint in the emergency department. Isolated facial edema is usually associated with angioedema or venous obstruction but the actual cause of obstruction might not be straightforward.

The authors present a case of a 56 year old woman who presented to the emergency department with a 2 year history of episodic facial and right upper limb edema, with no palpable masses or any physical examination alterations. Further study revealed a right parapharyngeal mass suggestive of a pleomorphic adenoma. The patient underwent surgery with no complications. Follow-up at one year with no evidence of recurrence.

The approach to the patient with isolated facial edema is always challenging. The immediate differential diagnosis usually relies on angioedema or venous thrombosis. Parapharyngeal space tumors are rare and usually present as an intraoral or cervical mass which did not happen in our case. The facial edema as the major complaint can be explained by the tumor growth compressing adjacent structures along with the internal jugular vein thrombosis.

Parapharyngeal space tumors are rare and can be quite challenging especially when the patient presents with peculiar symptoms. This case highlights a peculiar and rare presentation of a tumor and the importance of clinical thinking in a patient with exquisite complaints.
\end{abstract}

Keywords: Facial edema, parotid gland tumor, pleomorphic adenoma.

\section{Introduction}

One of the most common complaints at the emergency department is peripheral edema. The etiologies vary from inflammatory as in angioedema, obstructive (venous or lymphatic) or secondary to systemic disease (for example infection, heart failure or nephrotic syndrome). The approach to the patient with isolated facial edema mainly covers the differential diagnosis between angioedema and venous obstruction ${ }^{1}$. Tumors of the parapharyngeal space can cause localized edema secondary to venous obstruction and local inflammatory changes. They are rare accounting for $0.5 \%$ of all head and neck masses. The most common types are the salivary gland tumors, usually benign, that present as a palpable lump or mass or with compressive symptoms such as cranial nerve neuropathy ${ }^{2-3}$. The use of MRI (magnetic resonance imaging) in the initial evaluation might be a good cost-effective approach ${ }^{4}$.

\section{Case presentation}

The authors report a case of a 56 year old woman who presented to the emergency department with a two year history of recurrent episodic facial edema. The episodes usually lasted two to three weeks, resolved spontaneously and were sometimes associated with right upper limb edema and non pruriginous erythema. She denied any fever, loss of appetite or weight, sudoresis nor had noticed any cervical mass, hoarseness, difficulty breathing or dysphagia.

Past medical history included an idiopathic pulmonary embolism 10 years before medicated with vitamine $\mathrm{k}$ antagonist for 1 year, dyslipidemia and depression. Usual medication included sinvastatin $40 \mathrm{mg} q d$, trazodone $150 \mathrm{mg} q$ d.

Initial physical examination was unremarkable. Studies revealed no anemia, normal white blood count, isolated creatinine kinase elevation with normal myoglobin, normal renal function tests, slight elevation of the hepatic liver enzymes with normal albumin and prothrombine time. Cervical and axillar doppler ultrasonography without any vascular stenosis or obstruction; normal thoracic X-ray. The patient was admitted in an Internal Medicine ward. After a thorough physical examination there was a subtle facial edema evident in the right side of the face with unremarkable oral and neurological examinations. Further studies revealed a hypothyroidism and elevated ESR. A cervicothoracic CT scan was performed and revealed a right parapharyngeal space lesion and right internal jugular vein thrombosis. A cervical MRI was then performed which revealed a mass measuring $36 \times 42 \times 34 \mathrm{~mm}$ of the right parapharyngeal space with intense T2 signal and central contrast enhancement, suggestive of a pleomorphic adenoma (figure $1 \mathrm{~A}$ and $\mathrm{B}$ ). The patient started levothyroxine and anticoagulation and underwent surgery with total removal of the mass. Histological evaluation confirmed the pleomorphic adenoma. One year follow up with no evidence of recurrence or new complaints.

\section{Discussion}

The approach to the patient with isolated facial edema is always challenging. It is of the utmost importance to undergo a thorough clinical history and physical examination especially when the complaints are subtle and not obvious in physical examination as happened in our patient. The clinical brainstorming can be quite demanding when evaluating a patient with isolated facial edema. The immediate differential diagnosis usually relies on angioedema or venous edema (from an obstruction as in superior vena cava syndrome) $)^{1}$. Both are almost always obvious during clinical examination which did not happen with our patient.

Parapharyngeal space tumors are rare and most clinicians will only see a small number during their career ${ }^{2}$. Most of 
the case series published report first symptoms to be an intraoral or cervical mass and cranial nerve neuropathy as the lesions may compress cranial nerves IX to $X \|^{2,3,5}$. Unusual presentations have been reported such as sleep obstructive apnoea ${ }^{6}$.

What makes this case unique is the absence of any of the usual clinical signs. Remarkably the only symptoms presented where episodic facial and upper arm edema which lasted for two years before a diagnosis was established. Even the physical examination did not contribute with any clue as the patient's physical examination was unremarkable.

Although, at first, the location of the tumor seemed too high to explain the relationship with the facial edema, the fact that it compresses adjacent structures as the pterygoid plexus together with the internal jugular vein thrombosis (which is responsible for most of the venous drainage of the face) might justify the patient's complaints. Due to the indolent growing of the tumor we supposed that the venous system was able to progressively use other venous collaterals ultimately draining to the external jugular vein. As for the episodic upper limb edema, we suppose that the internal jugular vein thrombosis could have, at some point, extended to the junction point with the subclavian vein resulting in a partial occlusion of the upper limb drainage and therefore originating the edema described by the patient. To our understanding this is the rationale for her fluctuating symptoms.

Since the patient did not have any visible masses able to be accessed by ultrasound evaluation, our approach involved an initial CT scan. In our center, the general protocol dictates an initial head and/or neck CT scan and then, if justified, a MRI examination is performed. Growing knowledge shows that MRI offers a convenient and effective method when evaluating a patient with facial edema. It offers superior tissue discrimination and allows for a correct diagnosis of head and/or neck neoplasm and most inflammatory diseases ${ }^{4}$. Although overall it might appear cost-effective, we think that, in our reality, the major problem in using MRI as the first exam relies on availability and timely access.

Finally, as most of these tumors are diagnosed at a late stage we would like to raise awareness for subtle symptoms that can be the initial presentation of these tumors.

\section{Conclusion}

Parapharyngeal space tumors are rare and are usually diagnosed when a visible or palpable mass is obvious ${ }^{2,6}$. In patients presenting with peculiar symptoms such as episodic facial edema, the process of differential diagnosis can be quite challenging. This case highlights a rare presentation of a tumor and the importance of clinical thinking in a patient with exquisite complaints.
Figure 1. MRI image showing the right parapharyngeal mass (A) with hyperssignal in T2 and central contrast enhancement (B).

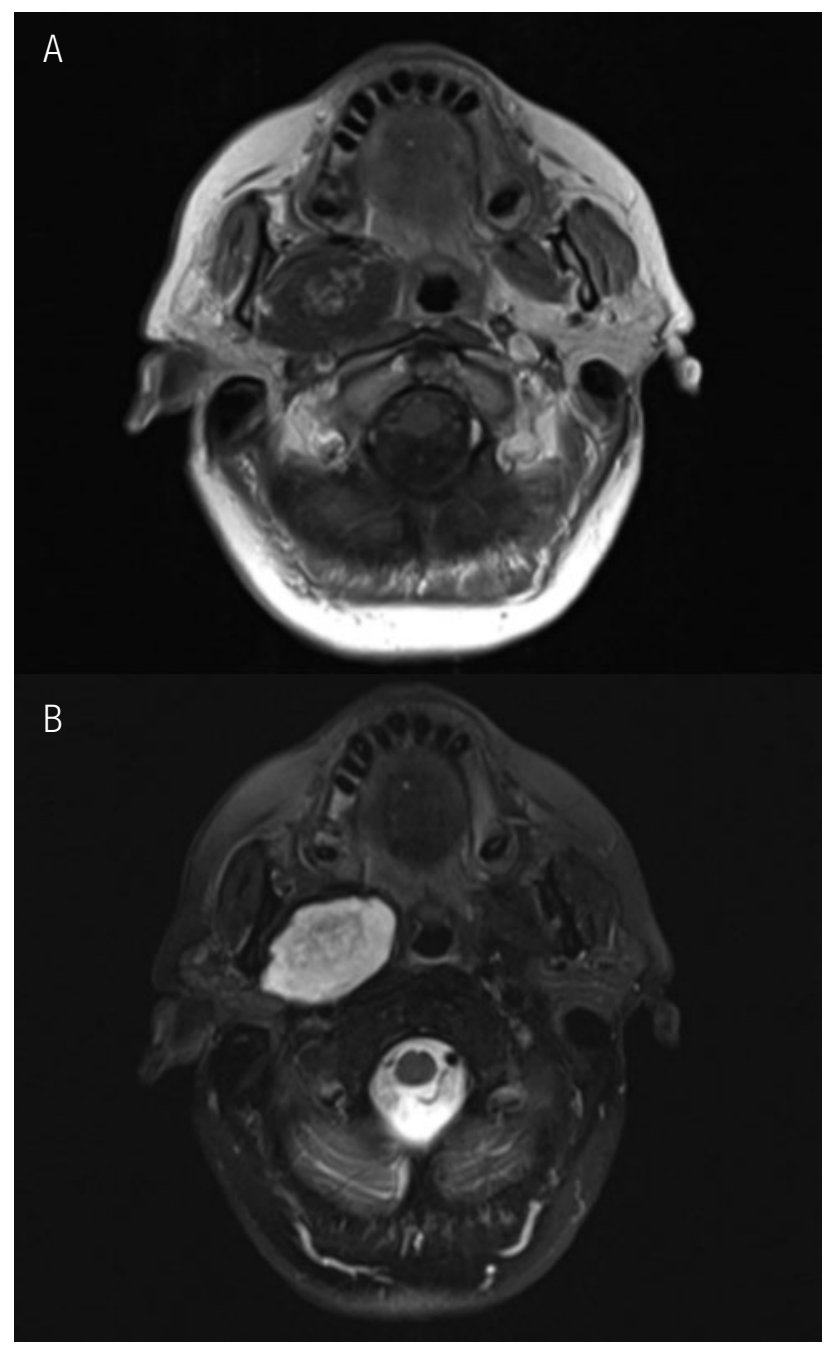

\section{References}

1. Siegenthaler W. Differential Diagnosis in Internal Medicine: From Symptom to Diagnosis. Edema. Stuttgart, Thieme. 2007, p 380-391.

2. Riffat F, Dwivedi RC, Palme C. Fish B, Jani P. A systematic review of 1143 parapharyngeal space tumors reported over 20 years. Oral Oncol. 2014; 50(5):421-30.

3. Lingam RK, Daghir AA, Nigar E, Abbas SA, Kumar M. Pleomorphic adenoma (benign mixed tumour) of the salivary glands: its diverse clinical, radiological, and histopathological presentation. Br J Oral Maxillofac Surg. 2011; 49(1):14-20.

4. Browne RF, Golding SJ, Watt-Smith SR. The role of MRI in facial swelling due to presumed salivary gland disease. Br J Radiol. 2001 Feb;74(878):127-33

5. Infante-Cossio P, Gonzalez-Cardero E, Gonzalez-Perez LM, Leopoldo-Rodado M, Garcia-Perla A, Esteban F. Management of parapharyngeal giant pleomorphic adenoma. Oral Maxillofac Surg. 2011; 15(4):211-6.

6. Wang AY, Wang JT, Levin B, Alam M, Palme CE, Riffat F. Parapharyngeal pleomorphic adenoma as a cause of severe obstructive sleep apnoea. ANZ J Surg. 2014;84(11):883-4. 